\section{From the NIH: A Systems Approach to Increasing the Diversity of the Biomedical Research Workforce}

\author{
Hannah A. Valantine, ${ }^{* *}$ P. Kay Lund, ${ }^{\dagger}$ and Alison E. Gammie ${ }^{\ddagger}$ \\ 'Office of the Director and ₹National Institute of General Medical Sciences, National Institutes of \\ Health, Bethesda, MD 20892
}

\begin{abstract}
The National Institutes of Health (NIH) is committed to attracting, developing, and supporting the best scientists from all groups as an integral part of excellence in training. Biomedical research workforce diversity, capitalizing on the full spectrum of skills, talents, and viewpoints, is essential for solving complex human health challenges. Over the past few decades, the biomedical research workforce has benefited from NIH programs aimed at enhancing diversity. However, there is considerable room for improvement, particularly at the level of independent scientists and within scientific leadership. We provide a rationale and specific opportunities to develop and sustain a diverse biomedical research workforce through interventions that promote the successful transitions to different stages on the path toward completion of training and entry into the biomedical workforce.
\end{abstract}

\section{A CALL TO ACTION}

The biomedical research career trajectory from trainee to independent scientist is not a linear pipeline but rather a series of opportunities and choices more aptly represented as pathways with branches (blogs.scientificamerican.com/voices/beyond -the-pipeline-reframing-science-s-diversity-challenge). Transition points along this career path offer a target to prevent the loss of highly trained scientists. For example, females make up more than half of biomedical science undergraduate (58\%) and postgraduate (53\%) degrees but only $18 \%$ of full professors in the biomedical sciences (www.nces.ed.gov/programs/digest/2013menu_tables.asp; www.nsf.gov/statistics/ wmpd/2013/pdf/nsf13304_digest.pdf). In contrast, males from racial/ethnic backgrounds that are well represented in science, technology, engineering, and mathematics (STEM) fields (i.e., white and Asian) make up 35\% of all undergraduate biomedical undergraduate degrees, $43 \%$ of all biomedical doctorates, and more than $82 \%$ of all full professorships (Figure 1). A striking loss of women and men from underrepresented backgrounds is apparent across the career spectrum (Figure 1). The diversity-focused efforts of funding agencies, including the National Institutes of Health (NIH), have contributed to an increase in $\mathrm{PhD}$ scientists from underrepresented groups; however, the low representation among faculty at academic institutions remains largely unchanged (Figure 1). Recent studies on career choices of graduate students reveal a significant attrition from biomedical research careers at rates that disproportionally affect women and underrepresented individuals (www.nap.edu/ catalog/12983/research-training-in-the-biomedical-behavioral-and-clinical -research-sciences; https://grants.nih.gov/training/minority_participation.pdf; http:// acd.od.nih.gov/diversity\%20in \%20 the \%20biomedical $\% 20$ research $\% 20$ workforce\%20report.pdf; Sauermann and Roach, 2012; Gibbs et al., 2014, 2015).

NIH has articulated four challenges that confront us: 1 ) the need for evidence-based recruitment and training, 2) further exploration of the impact of psychosocial factors on individual and institutional climate, 3) more study of the science of workforce
CBE Life Sci Educ September 1, 2016 15:fe4 DOI:10.1187/cbe.16-03-0138

*Address correspondence to: Hannah A. Valantine (hannah.valantine@nih.gov).

(c) 2016 H. A. Valantine et al. CBE-Life Sciences Education @ 2016 The American Society for Cell Biology. This article is distributed by The American Society for Cell Biology under license from the author(s). It is available to the public under an Attribution-Noncommercial-Share Alike 3.0 Unported Creative Commons License (http://creativecommons.org/licenses/ by-nc-sa/3.0)

"ASCB®" and "The American Society for Cell Biology $\AA^{\circledR}$ are registered trademarks of The American Society for Cell Biology. 


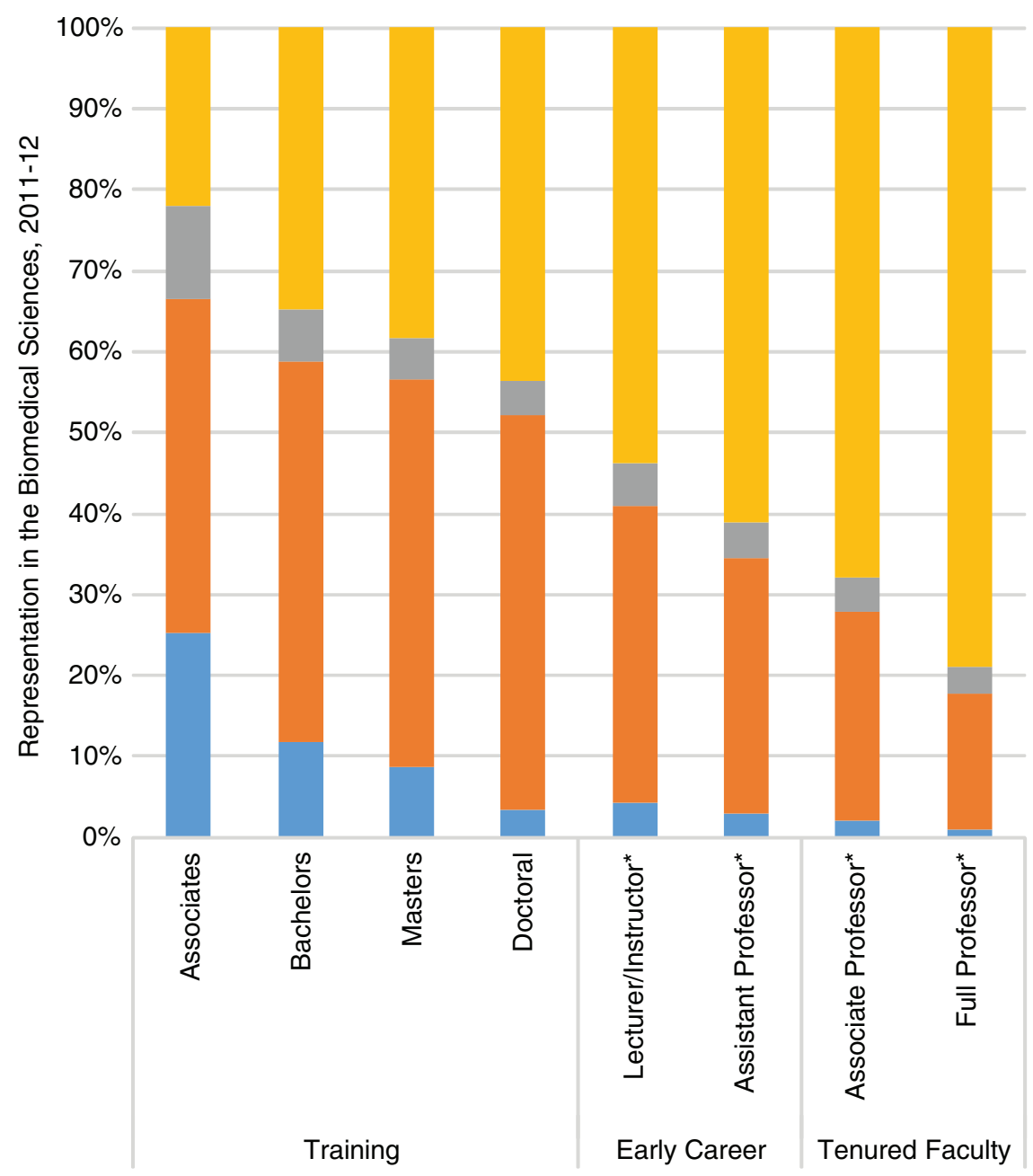

-Women - Underrepresented $\square$ Women - Well-represented

Men - Underrepresented $\square$ Men - Well-represented

FIGURE 1. Percent of underrepresented and well-represented trainees across career stages in the biomedical research workforce. Data represent the 2011-2012 academic year. Data were compiled using assessments from the 2013 Digest of Education Statistics (U.S. Department of Education, Institute of Education Sciences, National Center for Education Statistics [NCES], http://nces.ed.gov/programs/digest/2013menu _tables.asp). Gender and racial/ethnicity data analyzed by academic field (i.e., biological and biomedical sciences) at each stage of the academic training/career pathway. Scientists from underrepresented groups include blacks/African Americans, Hispanics, Native Americans and Alaska Natives, Pacific Islanders, and multiracial scientists. Scientists from well-represented groups include whites, Asians, and non-U.S. residents. While gender and racial/ethnicity data from the 2013 NCES Digest are disambiguated by academic field during the training stages (i.e., trainees awarded biomedical science degrees at the associate's through doctoral stages) in the 2011-2012 academic year, similar disambiguation of data is not currently available for the 2011-2012 academic year for early-career and tenured faculty. Instead, data were compiled from multiple NCES data sets to estimate gender and racial/ethnicity representation in the biomedical research and teaching workforce (refer to * in figure). Initial 2011-2012 data pertaining to gender and racial/ethnicity representation in the total professoriate across all academic career stages were adjusted to reflect differences in gender and racial/ethnicity representation between the total professoriate and that of the biomedical professoriate. These estimated 2011-2012 findings were further substantiated by close alignment with disambiguated 2004 NCES Digest findings. diversity, and 4) a national strategy to sustain workforce diversity in academia and beyond (Valantine and Collins, 2015). Our shared vision is to enhance successful transition from trainee to independent biomedical researcher for individuals from all groups, especially those currently underrepresented. Integrated and coordinated efforts across NIH provide an unprecedented opportunity to enhance biomedical research workforce diversity more rapidly and significantly.

\section{MAPPING THE NIH TRAINING AND DIVERSITY INVESTMENT}

In 2014, NIH appointed Dr. Hannah A. Valantine as chief officer for scientific workforce diversity (COSWD), a position that reports directly to the NIH director. The COSWD's charge is to enhance diversity of the biomedical research workforce through evidence-based research and to better coordinate and ensure efficacy of diversity initiatives across NIH. Dr. Kay Lund heads the newly created Division of Biomedical Research Workforce (DBRW), in the NIH Office of Extramural Research, which is tasked with effective implementation of policies to train, sustain, enhance, and increase diversity of the future biomedical workforce (http://acd.od.nih .gov/biomedical_research_wgreport.pdf). DBRW uses in-depth research, evaluation, economic analyses, and modeling to guide development of long-term policies and extramural programs focused on training and career development for biomedical research careers in academia, industry, and other research-related occupations. Valantine and Lund cochair the Federal Coordination in STEM Education Broadening Participation and Graduate Education interagency working groups, whose goals are to promote communication across agencies toward sustaining and diversifying the biomedical workforce.

Dr. Alison Gammie, the new director of the Division of Training Workforce Development and Diversity within the National Institute of General Medical Sciences (NIGMS/TWD) plays an integral role in the NIH commitment to scientific workforce diversity. NIGMS supports nearly half of all NIH-funded predoctoral trainees and a quarter of all trainees who receive $\mathrm{NIH}$ funding. NIGMS/TWD is strongly committed to training underrepresented individuals through a variety of programs at all stages of the career development 


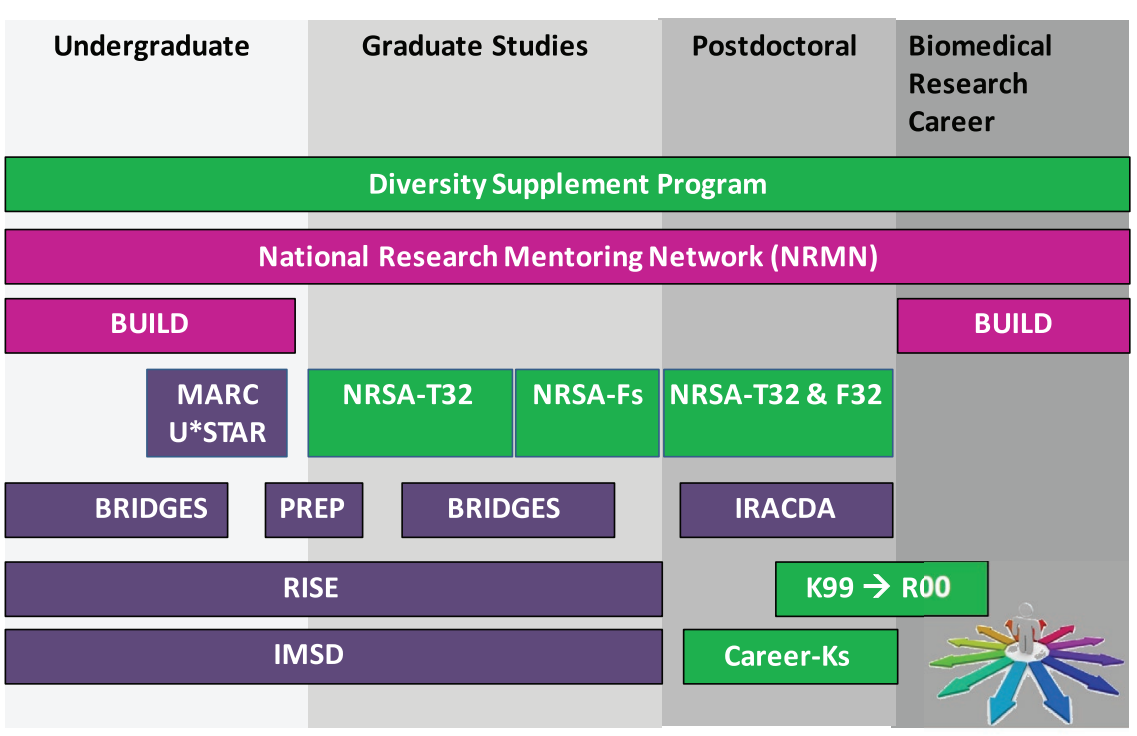

FIGURE 2. NIH biomedical research training programs. Schematic of NIH programs that fund trainees at the undergraduate, graduate, postdoctoral, and early biomedical research career stages that include: Research Supplements to Promote Diversity in Health-Related Research (Diversity Supplement Program), National Research Mentoring Network (NRMN), Building Infrastructure Leading to Diversity (BUILD), MARC Undergraduate Student Training in Academic Research (MARC U*STAR), Bridges to the Baccalaureate (BRIDGES), Postbaccalaureate Research Education Program (PREP), Bridges to the Doctorate (BRIDGES), Research Initiative for Scientific Enhancement (RISE), Initiative for Maximizing Student Development (IMSD), Institutional Predoctoral National Research Service Award (NRSA-T32), Individual Predoctoral National Research Service Award Fellowships (NRSA-Fs), Institutional Postdoctoral National Research Service Award (NRSA -T32), Individual Postdoctoral National Research Service Award (NRSA- F32), Institutional Research and Academic Career Development Award (IRACDA), Pathway to Independence Award (K99/R00), and Career Development Awards (Career-Ks). Purple, NIGMS programs; green, programs offered by multiple NIH institutes and centers; magenta, NIH Common Fund program.

pathway, complementing other programs offered by NIH institutes and centers (Figure 2). The relative numbers of registered trainees and early-career scientists supported across NIH are shown in Figure 3. This figure illustrates that efforts to diversify the workforce are focused on early-career stages, and less so on later stages in the pathway. The figure thus underscores the need for effective diversity efforts throughout the entire pathway to ensure successful transition from training into a biomedical workforce that encompasses a range of careers essential to our nation's scientific enterprise.

Several current NIH interventions already target key career transition points. For example, all NIH institutes and centers offer diversity supplements to support individuals from underrepresented groups or disadvantaged backgrounds to engage in research (http://grants.nih.gov/grants/guide/pa-files/PA-15 -322.html). Since 1992, NIH has awarded more than 1000 diversity supplement awards every year to multiple types of active grants and across the entire career spectrum. In 2014, awards were made at the high school (2\%), undergraduate (4\%), postbaccalaureate/master's (11\%), predoctoral (43\%), postdoctoral (30\%), and investigator (10\%) levels. Supplements to engage or retain investigators may be particularly important, given recent evidence that individuals at this stage are especially likely to leave the workforce (Dzirasa et al., 2015;
Heggeness et al., 2016). To address this career transition, multiple NIH institutes (National Cancer Institute, National Heart, Lung, and Blood Institute, National Institute of Neurological Disorders and Stroke) offer individual mentored career development awards (referred to with the prefix "K") to promote faculty diversity. Another example of an intervention at a key transition point is the NIGMS Institutional Research and Academic Career Development Award (IRACDA) program. IRACDA combines a traditional, mentored postdoctoral research experience with an opportunity to develop academic skills, including teaching, through workshops and mentored teaching assignments at a partner institution. This long-standing program has strong participation of women and underrepresented individuals and a high proportion of scholars attaining faculty positions (data forthcoming from NIGMS).

\section{A SYSTEMS APPROACH: MODULATING INPUTS AND OUTPUTS}

Our ability to achieve lasting diversity across the career spectrum will benefit from data-driven systems approaches that link the NIH investment in training (inputs) with measurable outcomes (outputs). A systems approach requires that we understand how outcomes at each career stage are influenced by institutional settings and interventions and by individual circumstances and decisions.

Acting on recommendations of the NIH Advisory Committee to the Director's working group report on diversity in the biomedical research workforce, and in the wake of the Ginther report documenting disparities in NIH R01 funding for African-American scientists (Ginther et al., 2011), NIH launched an experimental program in 2014 to implement and assess evidence-based practices in recruitment and retention of underrepresented students while simultaneously building infrastructure and developing faculty. The $\$ 250$ million, five-year Diversity Program Consortium (DPC; www.diversityprogramconsortium .org) consists of three integrated elements: the Building Infrastructure Leading to Diversity (BUILD) program; the National Research Mentoring Network (NRMN); and a Coordination and Evaluation Center. This experimental set of awards is providing data to help shape and revise current diversity training programs and inform new ones.

Several recent studies illustrate effective methods to quantify psychosocial quotients such as culture, prestige, bias, and stereotype threat that have a significant impact on why people join, stay, or leave science (Westring et al., 2012; Carnes et al., 2015; Clauset et al., 2015; Fassiotto et al., 2016). The DPC explicitly addresses the concepts of unconscious bias and stereotype threat. Other recent NIH-funded research has 


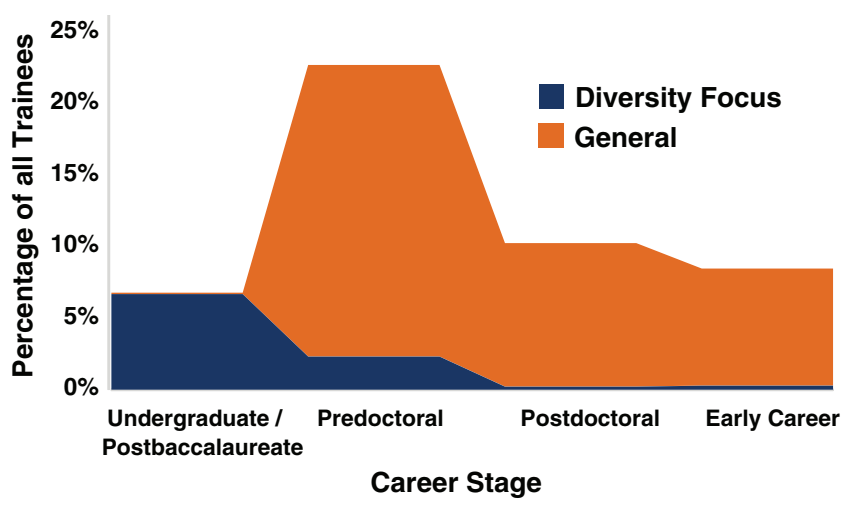

FIGURE 3. Estimate of Fiscal Year 2015 NIH training/career development participants by career state and diversity focus. An estimate of the percentage of trainees and early-career scientists in 2015 being supported by NIH through traditional training/career development programs (orange) and diversity-focused training/ career development programs (blue); data represent participants in the F/T/K module in the NIH Query, View, and Report system, which incorporates individual fellowships/mentored awards as part of the records, as well as appointees currently available in the NIH xTrain reporting system. Approximately $4 \%$ of trainees and early-career scientists were not trackable and thus are not mapped onto the career pathway. Diversity Supplement Program participants and participants in the Loan Repayment Program were not included in this estimate. In cases in which programs addressed multiple career stages of a trainee's career, proportions of trainees/ early-career scientists were assigned to each category based on estimates of the distribution of training/career development participant types in that program. Note that, although the figure depicts continuous data in a line graph, the assumption is that there are no intermediate percentages of trainees funded between career stages.

identified measures and interventions to address cultural barriers and institutional climate, including negative influences such as bias (implicit and explicit) and stereotype threat. These studies are built on social science theories, such as communities of practice, cultural capital, and social cognitive career theory (Thakore et al., 2014). One BUILD-funded institution uses critical race theory (Rosso, 2005) to frame its study and intervention on factors that influence students' decisions to persist in science careers (www.csun.edu/ build-poder).

A growing body of research on mentoring is instructive for designing and implementing programming that may have a positive impact on transition from training to employment or independence. For example, a recent NIH-funded randomized controlled trial (RCT) conducted across several academic medical centers showed that systematic, formalized, competency-based research mentor training improved self-reported mentor skills among mentors and mentoring behavior (Pfund et al., 2014). This work built upon earlier NIH-funded research that established the Mentoring Competency Assessment, a 26-item skills inventory that enables mentors and mentees to assess various competencies, including communication, expectations, diversity, independence, and professional development (Fleming et al., 2013). Another NIH-funded RCT involving individuals from underrepresented groups showed positive, albeit short-term, benefit from mentor training on mentees' satisfaction with mentors (Lewis et al., 2015). We hope to learn more from the NRMN, an innovative laboratory space for analyzing mentoring approaches in varied institutional contexts.

Increasingly, graduate students and postdoctoral trainees have been opting for careers outside academia (Fuhrmann et al., 2011; Sauermann and Roach, 2012; Stephan, 2012). NIH supports the Broadening Experiences in Scientific Training (BEST) program, which funds development of innovative approaches to complement traditional research training in biomedicine. Currently, BEST funds 17 institutions to explore novel interventions to enhance trainees' awareness, preparation, and skill acquisition for the opportunities in multiple biomedical research career pathways within and beyond academia. Effective partnerships with nonacademic entities are a key facet for implementation and evaluation.

Looking to the future, nascent "NIH Hubs of Innovation" intend to involve public-private partnerships working toward a shared goal of eliminating transition barriers along the biomedical career path and creating rapid, sustainable transformation in scientific workforce diversity. The Hubs of Innovation concept will establish multisector networks involving a range of institution types within each network, including tech, biotech, pharma, policy and academia-both research-intensive and teaching, and minority- and majority-serving. Each hub will be driven by an interdisciplinary program design team with expertise in biomedicine, technology, social science, business, health systems, and organizational effectiveness. Using sound research and design principles, hubs will aim to create, deploy, test, and disseminate new prototype diversity programs designed to meet the needs of sectors that are essential for biomedicine and biomedical research. Grounded in research and leveraging the diversity of expertise among its network members, hubs will aim to produce evidence-based strategies and programs to more rapidly diversify the biomedical workforce at all levels. An early target of each hub will be enhancing the diversity among researchers transitioning from training stages into the independent biomedical research workforce.

\section{CONCLUSION}

There is no doubt that today's America looks very different from that of 1930, when NIH was established. Major shifts in U.S. demographics speak volumes about society and our nation, and the changes demand a broader diversity of scientific talent in laboratories, classrooms, boardrooms, courts, think tanks, and the many other places where 21st-century scientists are employed. Further enhancement of diversity in the biomedical workforce, especially at the transitions to independence and leadership, is essential and doable. Achieving this goal requires a mind-set shift, from pipeline-based thinking to viewing biomedical research workforce diversity as a dynamic system. We can build immediately on NIH's biomedical training investment through more focus on the system's nodes where attrition is most common. Stated explicitly, that means focusing on retention, continuity, flexibility, and innovation across branch points in the career pathway. Doing so will benefit NIH and its stakeholders and also open the gates for highly trained scientists from diverse backgrounds to join the full range of careers needed to make robust contributions to the biomedical research enterprise. 


\section{ACKNOWLEDGMENTS}

We thank Alison Davis for editorial assistance in writing the manuscript and Andrew Miklos and Brad Newsome for data analyses.

\section{REFERENCES}

Carnes M, Devine PG, Baier Manwell L, Byars-Winston A, Fine E, Ford CE Forscher P, Isaac C, Kaatz A, Magua W, et al. (2015). The effect of an intervention to break the gender bias habit for faculty at one institution: a cluster randomized, controlled trial. Acad Med 90, 221-230.

Clauset A, Arbesman S, Larremore DB (2015). Systematic inequality and hierarchy in faculty hiring networks. Sci Adv 1, e1400005.

Dzirasa K, Krishnan RR, Williams RS (2015). Incubating the research independence of a medical scientist training program graduate: a case study. Acad Med 90, 177-179.

Fassiotto M, Hamel EO, Ku M, Correll S, Grewal D, Lavori P, Periyakoil VJ, Reiss A, Sandborg C, Walton G, et al. (2016). Women in academic medicine: measuring stereotype threat among junior faculty. J Womens Health (Larchmt) 25, 292-298.

Fleming M, House S, Hanson VS, Yu L, Garbutt J, McGee R, Kroenke K, Abedin Z, Rubio DM (2013). The Mentoring Competency Assessment: validation of a new instrument to evaluate skills of research mentors. Acad Med 88 1002-1008.

Fuhrmann CN, Halme DG, O'Sullivan PS, Lindstaedt B (2011). Improving graduate education to support a branching career pipeline: recommendations based on a survey of doctoral students in the basic biomedical sciences. CBE Life Sci Educ 10, 239-249.

Gibbs K Jr (2014, December 17). Beyond "the pipeline": reframing science diversity's challenge [blog post] blogs.scientificamerican.com/voices/ beyond-the-pipeline-reframing-science-s-diversity-challenge (accessed 20 June 2016).
Gibbs KD Jr, McGready J, Bennett JC, Griffin K (2014). Biomedical science Ph.D. career interest patterns by race/ethnicity and gender. PLoS One 9, e114736.

Gibbs KD Jr, McGready J, Griffin K (2015). Career development among American biomedical postdocs. CBE Life Sci Educ 14, ar44.

Ginther DK, Schaffer WT, Schnell J, Masimore B, Liu F, Haak LL, Kington R (2011). Race, ethnicity, and NIH research awards. Science 333 1015-1019.

Heggeness ML, Evans L, Pohlhaus JR, Mills SL (2016). Measuring diversity of the NIH-funded workforce. Acad Med., doi: 10.1097/ACM.0000000000001209.

Lewis V, Martina CA, McDermott MP, Trief PM, Goodman SR, Morse GD, LaGuardia JG, Sharp D, Ryan RM (2015). A randomized controlled trial of mentoring interventions for underrepresented minorities. Acad Med, doi: 10.1097/ACM.0000000000001056

Pfund C, House SC, Asquith P, Fleming MF, Buhr KA, Burnham EL, Eichenberger Gilmore JM, Huskins WC, McGee R, et al. (2014). Training mentors of clinical and translational research scholars: a randomized controlled trial. Acad Med 89, 774-782.

Rosso TJ (2005). Whose culture has capital? A critical race theory discussion of community cultural wealth. Race Ethnic Educ 8, 69-91.

Sauermann H, Roach M (2012). Science PhD career preferences: levels, changes, and advisor encouragement. PLoS One 7, e36307.

Stephan PE (2012). How Economics Shapes Science, Cambridge, MA Harvard University Press.

Thakore BK, Naffziger-Hirsch ME, Richardson JL, Williams SN, McGee R Jr (2014). The Academy for Future Science Faculty: randomized controlled trial of theory-driven coaching to shape development and diversity of early-career scientists. BMC Med Educ 14, 160

Valantine HA, Collins FS (2015). National Institutes of Health addresses the science of diversity. Proc Natl Acad Sci USA 112, 12240-12242.

Westring AF, Speck RM, Sammel MD, Scott P, Tuton LW, Grisso JA, Abbuhl S (2012). A culture conducive to women's academic success: development of a measure. Acad Med 87, 1622-1631. 
H. A. Valantine et al.

\section{HIGHLIGHTS:}

The National Institutes of Health presents a systems approach to developing and sustaining a diverse biomedical workforce through interventions that promote successful transitions from training to career independence. 